\title{
Abandono, rendimiento académico y tutoría: una investigación de la Universidad de Padua
}

\section{Dispersione universitaria, rendimento accademico e tutorato: un'indagine dell'Università di Padova}

\section{Drop-out, performance and tutoring: an investigation at the University of Padua}

\author{
Lorenza Da Re ${ }^{1}$ \\ lorenza.dare@unipd.it \\ Renata Clerici \\ renata.clerici@unipd.it \\ Universitá degli Studi di Padova, Italia
}

\begin{abstract}
Riassunto:
L'Università di Padova ha recentemente affiancato, in via sperimentale, alle tradizionali azioni di tutorato, un nuovo Programma di Tutorato Formativo, con la finalità di promuovere il successo accademico degli studenti e contrastare il drop-out universitario, attraverso il rafforzamento di alcune competenze trasversali particolarmente utili nel percorso di studio. Al fine di definire il profilo degli studenti potenziali fruitori del programma, di rilevare le caratteristiche del drop-out e del rendimento in specifici contesti, e infine, di valutare la conoscenza e l'utilizzo dei servizi di tutorato, è stata progettata e condotta un'indagine Computer-Assisted Web Interviewing su una coorte di 754 studenti immatrico-
\end{abstract}

1 Dirección para correspondencia (correspondence address):

Lorenza da Re. Dipartimento di Filosofia, Sociologia, Pedagogia e Psicologia Applicata - FISPPA, Università degli Studi di Padova. P.zza Capitaniato, 3. 35139 Padova (Italia) 
Abandono, rendimiento académico y tutoría: una investigación de la Universidad de Padua

Lorenza Da Re y Renata Clerici

encuesta web en una cohorte de 754 estudiantes matriculados en el año académico 2012/13 en 3 grados elegidos como casos de estudio para la experimentación. En este artículo se presenta el instrumento y los indicadores utilizados para la recogida de información y algunos resultados que se consideran de particular interés para la aplicación del Programa.

\section{Palabras clave:}

Tutoría; abandono; rendimiento académico; educación superior; cuestionario. latisi nell'anno accademico 2012/13 in 3 corsi universitari di primo livello scelti come casi studio per la sperimentazione. In questo contributo presenteremo lo strumento e gli indicatori utilizzati per la rilevazione, e alcuni risultati ritenuti di particolare interesse per la realizzazione del Programma.

\section{Parole chiave:}

Tutorato; abbandono; rendimento accademico; educazione superiore; indagine.

\begin{abstract}
:
The University of Padua has recently integrated usual tutoring actions with a new experimental formative tutoring program. It aims to improve students' academic success and to reduce university drop-out, by means of reinforcing soft skills, which are particularly useful for the career of a student. A computer-assisted web interviewing survey was designed in order to define the profile of potential users of the program, to observe-in various academic contexts - the characteristics of the drop-out phenomenon and of student performance, and finally, to assess knowledge and use levels of tutoring services. The survey involved 754 students, enrolled during 2012-13 academic year in three first-level degree courses, which were selected as case studies for the experiment. In this study we present the survey questionnaire, the indicators used for data collection, and some results that we consider of particular interest for the implementation of the program.
\end{abstract}

\title{
Key words:
}

Tutoring; drop-out; academic performance; higher education; survey.

\section{Résumé:}

L'Université de Padoue a récemment integré, à titre expérimental, aux actions traditionnelles de tutorat, un nouveau programme de formation de tutorat, dans le but de promouvoir le succès et la réussite scolaire des élèves et contrer le drop-out (abandon) universitaire, par le renforcement de certains soft skills particulièrement utiles dans le programme d'études. Afin de définir le profil des utilisateurs potentiels des étudiants du programme, de détecter les caractéristiques de l'abandon et des performances dans des contextes spécifiques, et enfin, d'évaluer la connaissance et l'utilisation des services de tutorat, il a été conçu et mené une enquête intitulée Computer-Assisted Web Interviewing sur un groupe de 754 étudiants qui se sont inscrits dans l'année scolaire 2012/13 en trois cours universitaires de premier niveau choisi comme études de cas pour la expérimentation. Dans cet article nous allons présenter l'instrument et les indicateurs utilisés pour détecter et aussi certains résultats considérés d'intérêt particulier pour la mise en œuvre du Programme.

\section{Mots clés:}

Tutorat; abandon; performance académique; éducation supérieure; enquête.

\author{
Fecha de recepción: 24-2-2017 \\ Fecha de aceptación: 20-3-2017
}




\section{Introduzione}

Uno dei principali problemi delle Istituzioni Educative a livello mondiale riguarda il fenomeno della dispersione negli studi. Molti sono i lavori pubblicati su questa problematica che fanno emergere le dimensioni di un fenomeno preoccupante, che riguarda tutti i livelli scolastici. Al contempo, si sono andate sviluppando strategie educative che intendono rispondere a queste emergenze. Tra quelle segnalate in letteratura come particolarmente atte a contrastare e prevenire il drop-out, potenziare il rendimento accademico, supportare lo studente nelle scelte accademiche e professionali, figura il modello spagnolo di tutoría formativa de carrera (Álvarez, 2002).

L'Università di Padova, a partire dall'anno accademico 2014-15, ha dato il via a una sperimentazione di interventi di Tutorato Formativo (Da Re, 2016; Da Re, Álvarez e Clerici, 2015; Da Re, Clerici e Álvarez, 2016). Fondandosi sulle direttrici pedagogiche del modello spagnolo, è stato progettato un piano d'azione con attività di orientamento e tutorato per gli studenti di alcuni Corsi di Laurea Triennale, al fine di agevolare la transizione educativa dalla Scuola Secondaria all'Università, di potenziare alcune competenze trasversali particolarmente utili per lo specifico contesto, e di accompagnare lo studente nel suo percorso accademico. Per realizzare una opportuna selezione e un adeguato adattamento degli interventi e delle attività di Tutorato Formativo da proporre per il contesto italiano, è stata progettata e condotta un'indagine CAWI (computerassisted web interviewing) tra gli studenti dei Corsi di Studio (CdS) in cui è stata avviata la sperimentazione. Procedure e risultati di tale indagine saranno oggetto di questo articolo.

\section{Quadro teorico}

La letteratura che tratta il tema del drop-out è assai ricca di categorizzazioni e di schemi interpretativi, per cercare di dar risposte coerenti e di individuare soluzioni efficaci. Lo studio dei fattori che influiscono sull'abbandono e sul ritardo nei percorsi universitari ha consentito lo sviluppo di modelli esplicativi che individuano le principali determinanti del fenomeno e le variabili a esso connesse.

Se pur l'intenzionalità che porta a creare modelli interpretativi del 
Abandono, rendimiento académico y tutoría: una investigación de la Universidad de Padua

Lorenza Da Re y Renata Clerici

drop-out, e in generale della dispersione e dell'insuccesso nei percorsi di studio universitari, sia differente da Autore ad Autore, tutti fanno comune riferimento a fattori di carattere sia soggettivo (dello studente) che di contesto (dell'organizzazione universitaria).

Possiamo così categorizzare i fattori soggettivi in:

- Fattori caratterizzanti: differenze di genere (Arias Ortiz e Dehon, 2013; Barefoot, 2004; Charlton et al., 2006; DesJardins et al., 2002; O'Neill et al., 2011; Reason, 2009), di etnia (Arias Ortiz e Dehon, 2013; Belloc et al., 2010; Lyons, 2004), relative al luogo di residenza (Dolton et al., 2003; Smith e Naylor, 2001) e all'età all'immatricolazione (Belloc et al., 2010; Charlton et al., 2006).

- Fattori socio-culturali: legati alla famiglia di origine, alle condizioni economiche (Braunstein, Mc Grath, e Pescatrice, 2001; Cofer e Somers, 2001; Ishitani e Des Jardins, 2002), allo status di "studente lavoratore" (Dolton et al., 2003; Kim, 2007) e all'origine sociale (Latiesa, 1992).

- Fattori psicologici personali: bassa autostima (Masjoan, 1989), influenza dell'"ottimismo" (Seligman, 1990; Snyder, 2002), autoefficacia (Kirton, 2000), autoregolazione (Zimmerman, 2000), motivazione (Landry, 2003), altre caratteristiche psicologiche come la capacità di superare gli ostacoli e le difficoltà (Landry, 2003) e difficoltà pregresse o circostanze di vita (Sinclair e Dale, 2000).

- Fattori psico-pedagogici legati allo studio: difficoltà nelle strategie di apprendimento e numero di ore dedicate allo studio (Albalate et al., 2011).

- Fattori legati all'orientamento e alla scelta del percorso: errore nella scelta (Corominas, 2001) e peso dell'orientamento pre-universitario (Garcia Felix et al., 2014).

- Fattori legati alla formazione pre-universitaria: influenza del percorso pre-universitario (Soria-Barreto e Zúñiga-Jara, 2014), bocciature pre-universitarie (Arias Ortiz e Dehon, 2013), voto all'esame di maturità (DesJardins et al., 2002), cattiva formazione pre-universitaria (Zubieta e Susinos, 1986).

- Rendimento accademico effettivo: apprendimento non attivo e basse aspettative circa i risultati accademici (Landry, 2003).

I fattori del contesto, relativi all'organizzazione universitaria, possono essere così categorizzati: 
- Fattori riguardanti la struttura del CdS: vicinanza dalla sede (Dolton et al., 2003; Smith e Naylor, 2001), numero chiuso e frequenza alle lezioni (Albalate et al., 2011), area scientifico-didattica di riferimento (Arias Ortiz e Dehon, 2013; Patrick, 2001), dimensione del corso (Arulampalam et al., 2004; Kim, 2007; Ryan, 2004; Titus, 2004).

- Fattori riguardanti I'organizzazione universitaria e I'integrazione nel contesto universitario: integrazione nel contesto universitario (Landry, 2003; Metz, 2002; Smith e Naylor, 2001; Spady, 1970; Tinto, 1975), discrepanza tra aspettative iniziali e quelle reali (Metz, 2002), possibilità lavorative future (Luján e Resendiz, 1981).

- Fattori riguardanti i servizi offerti dall'Ateneo e dal CdS: conoscenza ed utilizzo dei Servizi dell'Ateneo, del CdS e del Tutorato (Pérez, Martínez e Martínez, 2015; Zago et al., 2014), scarse condizioni organizzative (Braxton e Mundy, 2002; Berger, 2002; Kuh, 2002; Montmarquette, Mahseredjian, e Houle, 2001; Thomas, 2002), soddisfazione del percorso di studio che si frequenta o insoddisfazione e poca utilità del CdS (Mashburn, 2000).

I fattori del contesto, relativi alla docenza e alla didattica, possono essere così categorizzati:

- Rapporto con i docenti: metodologia didattica (Arulampalam et al., 2004; Chen, 2012; Patrick, 2001; Thomas, 2002), supporto personalizzato agli studenti e tratti specifici del docente (Barefoot, 2004).

- Rapporto con le discipline: non superamento di esami particolarmente difficili (Escandell e Marrero, 1999).

Le teorie più accreditate sono quelle che individuano una multicausalità del problema (Clerici, Giraldo, e Meggiolaro, 2015; Forbes e Wickens, 2005; Kirton, 2000; Mashburn, 2000; Meggiolaro, Giraldo, e Clerici, 2015). Moxley, Najor-Durack, e Dumbrigue, 2001), quindi non solo un fattore, ma una pluralità di con-cause possono dare origine al problema.

Tra i fattori che possono influire sul percorso di studio, sottolineiamo l'importanza delle azioni di supporto per gli studenti, in particolare dai servizi universitari, intesi come quei supporti offerti a immatricolandi e studenti per creare un ambiente ideale per l'apprendimento. Tali servizi consentono allo 
Abandono, rendimiento académico y tutoría: una investigación de la Universidad de Padua

Lorenza Da Re y Renata Clerici

studente di accedere alla formazione universitaria nelle migliori condizioni per apprendere. Emerge da alcune ricerche (Fabbris, 2010) che lo studente oggi utilizza in maniera sempre più partecipe e attiva i servizi offerti dal sistema universitario. Gli Atenei, da parte loro, organizzano attività e servizi di supporto alla didattica e agli studenti in modo sempre più attento, in risposta alle esigenze degli studenti e delle singole realtà.

Tra questi, le azioni di orientamento e tutorato, applicate in ambito universitario (Álvarez, 2002; 2005; Da Re, Clerici e Álvarez, 2016; Da Re e Zago, 2014; Martìnez Clares et al., 2014; Pantoja, 2005; Zabalza, 2003), rivestono un importante ruolo per quanto riguarda l'integrazione e l'accompagnamento degli studenti nel nuovo contesto di apprendimento. Entrambi si propongono di prevenire e contrastare l'abbandono e il rallentamento degli studi universitari e di aiutare gli studenti ad attuare scelte consapevoli, sostenendoli in eventuali fasi critiche del percorso di studio e di vita (Da Re e Zago, 2014).

\section{Le domande di ricerca}

Sono state definite una serie di domande di ricerca volte a interpretare il successo e l'insuccesso nelle carriere universitarie, attraverso fattori individuali e contestuali osservati.

Queste domande sono esplicitate in tabella 1 e, rispettivamente, riferite alle azioni dei fattori individuali e dei fattori contestuali. I contenuti concettuali di ciascuna domanda di ricerca sono stati preliminarmente tradotti empiricamente attraverso indicatori e variabili desunti dai dati forniti sia dall'indagine che dagli archivi amministrativi dell'Università. Nella maggior parte dei casi, gli indicatori utilizzati sono stati costruiti come sintesi di più variabili.

Si è innanzitutto definito un indicatore di sintesi che esprimesse la situazione di carriera dello studente, sia in termini oggettivi (crediti effettivamente maturati) che soggettivi rispetto al percorso svolto (ritieni che il tuo percorso sia stato rallentato) e rispetto al futuro prossimo (di quanti anni pensi avrai bisogno per terminare gli studi). L'analisi delle componenti principali ha verificato l'esistenza e la forte associazione (Sig.=.000) tra due dimensioni concettuali, la prima definita "percezione attuale e aspettative di regolarità della carriera universitaria", che assorbe la maggior parte della variabilità (inerzia=.889), va dalla "regolarità" al "rallen- 
tamento", passando per il cambio di CdS, e la seconda "performance accademica" dello studente alla fine del primo anno di corso (inerzia=.024) che va dal "pessimismo" all'"ottimismo", passando per "rassegnati", "ottimisti non realisti", "realisti fiduciosi" e "ottimisti realisti".

Tabella 1. Domande di ricerca e indicatori utilizzati per l'operazionalizzazione.

(A) Fattori individuali (studente) Indicatori

DR1. Alcune caratteristiche socio-demografiche in- $\rightarrow$ Residenza fluenzano il rendimento accademico?

$\rightarrow$ Età

$\rightarrow$ Sesso

$\rightarrow$ Scuola di provenienza

DR2. La condizione socio-economica influenza il $\rightarrow$ Esperienze universitarie rendimento accademico? (fattori socio-culturali: in famiglia legati alla famiglia di origine e alle condizioni $\rightarrow$ Condizione lavorativa economiche)

$\rightarrow$ Benefici economici

DR3. Alcuni fattori psicologici legati allo studio in- $\rightarrow$ Capacità di studio fluenzano il rendimento accademico?

$\rightarrow$ Motivazione

DR4. Che influenza ha l'orientamento e la moti- $\rightarrow$ Scelta consapevole e vazione alla scelta nel rendimento? (fattori legati alla consapevolezza della scelta fatta per ragionata I'Università e per il CdS specifico).

DR5. Il percorso pre-universitario influenza il rendi- $\rightarrow$ Regolarità del percorso mento accademico? (fattori legati alla formazio- pre-universitario ne pre-universitaria: scarso esito nella scuola su- $\rightarrow$ Voto di diploma periore, bocciature, esito all'esame di maturità).
(B) Fattori del contesto (sistema universitario)
Indicatori

DR6. Alcuni fattori contestuali influenzano il per- $\rightarrow$ Sede di studio e domicorso di studio? (il pendolarismo? La frequenza alle lezioni?) cilio $\rightarrow$ Frequenza lezioni

DR7. Conoscere e utilizzare i servizi è predittivo $\rightarrow$ Conoscenza/utilizzo tudel successo? (fattori riguardanti i servizi offerti torato dall'Ateneo e dal CdS: conoscenza ed utilizzo; $\rightarrow$ Conoscenza dei servizi nello specifico il tutorato).

DR8. La soddisfazione rispetto al percorso che si $\rightarrow$ Soddisfazione rapporti svolge è predittiva del successo o dell'insuccesso? con gli attori $\rightarrow$ Soddisfazione per CdS $\rightarrow$ Soddisfazione generale 
Abandono, rendimiento académico y tutoría: una investigación de la Universidad de Padua

Lorenza Da Re y Renata Clerici

(C) Fattori del contesto (didattica e docenza)

Indicatori

DR9. Alcune questioni legate alla didattica influis- $\rightarrow$ Valutazione della didatcono sul percorso di studio?

tica

DR10. Alcune questioni legate alla docenza in- $\rightarrow$ Valutazione della dofluiscono sul percorso di studio? cenza

\section{Metodo}

Lo studio di seguito presentato è relativo alla progettazione e alla conduzione di un'indagine CAWI su una coorte di 754 immatricolati nell'a.a. 2012/13 in alcuni selezionati Corsi di Studio dell'Università di Padova. Scopo dell'indagine era di rilevare le caratteristiche della dispersione universitaria in specifici contesti, e di misurare la conoscenza e l'utilizzo dei servizi di tutorato. L'obiettivo specifico era di rispondere a due principali quesiti di ricerca: (i) qual è il profilo degli studenti che abbandonano nei Corsi scelti come casi di studio?, (ii) gli studenti conoscono e utilizzano il Servizio Tutorato?

La popolazione di riferimento è costituita dagli immatricolati di tre Corsi di Studio triennali (Ingegneria Meccanica, Scienze dell'Educazione e della Formazione, Scienze Sociologiche), osservata a un anno circa dall'immatricolazione (cioè a metà del secondo anno di corso).

Il questionario utilizzato per la rilevazione dei dati è stato formulato ad hoc, anche attraverso una selezione ragionata di quesiti utilizzati in indagini nazionali e internazionali, in relazione a vari specifici obiettivi, al fine di definire uno strumento che, con opportuni aggiustamenti e correzioni, fosse rispondente alle finalità della presente ricerca.

Esso è composto da sei sezioni (1. Background, 2. Percorso di Studio, 3. Tutorato, 4. Docenza e didattica, 5. Atteggiamenti e opinioni, 6. Chiusura del questionario) e da sette Percorsi (regolare, abbandono, cambio di CdS - in uscita, cambio di Ateneo - in uscita, cambio di CdS - in entrata, cambio di Ateneo - in entrata, rallentamento).

I dati raccolti tra gli studenti mediante questa indagine diretta sono stati integrati da una serie di notizie che sono ricavate dagli archivi amministrativi di Ateneo. A partire da questi sono stati costruiti degli indici di sintesi, con l'obiettivo di rendere maggiormente evidenti le dimensioni concettuali dell'operativizzazione dei fattori rilevanti nella interpretazione dei fenomeni di interesse. 
La somministrazione è avvenuta online tramite LimeSurvey², per consentire agli studenti di poter valutare con calma le singole domande. Queste sono state caricate manualmente e sono stati impostati i rispettivi filtri, in funzione dei sette diversi percorsi ipotizzati. Per ogni singolo rispondente è stato costruito un Token, ossia un codice che funge da identificativo e che consente di sapere chi ha risposto pur garantendone I'anonimato.

Sono stati richiesti all'ufficio competente gli indirizzi degli studenti della coorte 2012, cioè immatricolati nell'anno accademico 2012-13. I dati richiesti comprendevano i nominativi dei partecipanti, la loro matricola, la e-mail istituzionale e la e-mail privata. L'uso di queste informazioni è stato funzionale al contatto e all'aggancio coi dati d'archivio, ma ricade nell'impegno di riservatezza assunto con i rispondenti a non diffondere, se non in forma aggregata, alcuna notizia personale. In primo luogo, sono stati "puliti" i dati d'archivio, togliendo chi non aveva esplicitamente dato I'assenso all'acceso ai propri dati: da 754 studenti siamo arrivati a 743.

\section{Risultati e discussione}

La popolazione di riferimento risulta costituita da 743 casi: 322 immatricolati al CdS in Ingegneria Meccanica (ING_MEC), 302 al CdS in Scienze dell'Educazione e della Formazione (SC_EF) e 119 al CdS in Scienze Sociologiche (SC_SOC).

I questionari compilati sono risultati 167 (55 di ING_MEC, 82 SC_EF, 30 SC_SOC). Il tasso di risposta è complessivamente del 22.5\%: il 17\% per Ingegneria Meccanica, 27\% per Scienze dell'Educazione e 25\% per Scienze Sociologiche. Una verifica della rappresentatività dei rispondenti rispetto alla popolazione di riferimento è stata condotta con riferimento a una serie di variabili associate ai fenomeni in analisi (tipo di scuola secondaria di provenienza, stato della carriera, provenienza geografica, età): per tutti e 3 i CdS analizzati il confronto non ha evidenziato differenze significative ${ }^{3}$.

2 LimeSurvey è un software open source dedicato alla implementazione di questionari CAWI.

3 Le analisi statistiche sono state condotte con il programma Statistical Package for the Social Sciences (SPSS 22.0) per Microsoft Windows 7. 
Abandono, rendimiento académico y tutoría: una investigación de la Universidad de Padua

Lorenza Da Re y Renata Clerici

La maggioranza dei rispondenti proviene da un Liceo (55\%) o da un Istituto Tecnico (35\%). II 96\% della popolazione è "attiva", cioè regolarmente iscritta, mentre il $4 \%$ ha cessato la carriera universitaria. II 92\% risiede in una provincia della Regione Veneto mentre I' $8 \%$ è residente fuori regione. L' $88 \%$ ha età compresa tra i 20 e 24 anni.

Gli studenti "regolari" costituiscono il 49\% dei rispondenti, il 35\% segnala un rallentamento del percorso; il $4 \%$ ha abbandonato gli studi e il $12 \%$ ha cambiato CdS. Questi livelli differiscono notevolmente nei 3 CdS analizzati. In particolare ING_MEC si caratterizza per un elevatissimo rallentamento $(47 \%)$.

In linea con le finalità della ricerca la sezione "tutorato" è stata approfondita con particolare cura. In riferimento alla conoscenza e all'utilizzo del tutorato dell'Università di Padova (Da Re e Zago, 2014), complessivamente il 57.4\% degli studenti rispondenti dichiara di conoscere ma non utilizzare il Servizio Tutorato, il $21.3 \%$ lo conosce e lo ha utilizzato e il $21,3 \%$ non lo conosce e non lo utilizza.

Dai dati (Tab. 2) emerge che la distribuzione dei livelli di conoscenza non è significativamente diversa tra i CdS (Sig.=.16): I'analisi evidenzia che gli studenti di Ingegneria Meccanica sono quelli che meno conoscono $(34 \%)$ e meno utilizzano $(47.2 \%)$ il Servizio Tutorato.

\section{Tabella 2. Percentuali dei rispondenti dei CdS per conoscenza e utilizzo del}

\section{Servizio Tutorato.}

Conoscenza e utilizzo del Servizio Tutorato

\begin{tabular}{cccccc}
\hline & Conosce e utilizza & $18.9 \%$ & $21.8 \%$ & $24.1 \%$ & $21.3 \%$ \\
& Conosce ma non utilizza & $47.2 \%$ & $67.9 \%$ & $48.3 \%$ & $57.5 \%$ \\
& Non conosce & $34 \%$ & $10.3 \%$ & $27.6 \%$ & $21.3 \%$ \\
Totale & & $100 \%$ & $100 \%$ & $100 \%$ & $100 \%$ \\
\hline
\end{tabular}

La modalità di contatto più efficace per ING_MEC risulta essere il passaparola (44.1\%), mentre per SC_EF le presentazioni in aula (44.6\%), infine, per SC_SOC il sito web $(60 \%)$, ma anche rilevante appare il ruolo del passaparola $(40 \%)$.

In riferimento al tipo di servizio ricevuto, gli studenti di ING_MEC dichiarano di avere ricevuto, nella maggioranza dei casi, orientamento in itinere (90\%) e proposte di gruppi studio (50\%); gli studenti di SC_SOC unicamente informazioni sulla didattica $(100 \%)$ e orientamento in itine- 
re $(100 \%)$; quelli di SC_EF principalmente informazioni sulla didattica $(73.3 \%)$ e orientamento in itinere $(73.3 \%)$.

Circa la valutazione degli aspetti relativi al Servizio Tutorato (da $1_{-}$ completamente insoddisfatto/a a 10_completamente soddisfatto/a) (Tab. 3), non emergono significative differenza nelle valutazioni medie dei vari aspetti per i diversi CdS. Si può comunque sottolineare come gli elementi che sono stati valutati generalmente in modo più positivo siano stati la disponibilità dei Tutor Junior ${ }^{4}$ (valore medio 7.8) e la modalità di contatto (valore medio 7.9). Da sottolineare come gli studenti di ING_MEC valutino in modo particolarmente positivo la pagina web dei Tutor Junior nel sito del CdS (valor medio 8).

Tabella 3. Soddisfazione media per il servizio tutorato (ANOVA tra CdS - Test F).

\begin{tabular}{|c|c|c|c|c|}
\hline Servizio Tutorato & CdS & $\begin{array}{c}\text { Soddisfazione } \\
\text { media }\end{array}$ & $\begin{array}{l}\text { Dev. } \\
\text { Stand. }\end{array}$ & Significatività \\
\hline \multirow{3}{*}{$\begin{array}{l}\text { In che misura ritieni che i Tu- } \\
\text { tor Junior ti abbiano aiutato/a } \\
\text { a risolvere la questione per } \\
\text { cui ti sei rivolto/a loro }\end{array}$} & $\begin{array}{l}\mathrm{ING}_{-} \\
\mathrm{MEC}\end{array}$ & 6.4 & 1.8 & \multirow{3}{*}{.930} \\
\hline & SC_EF & 6.7 & 2.1 & \\
\hline & SC_SOC & 6.9 & 2.6 & \\
\hline \multirow{3}{*}{$\begin{array}{l}\text { In che misura hai gradito il } \\
\text { modo in cui i Tutor Junior si } \\
\text { sono relazionati con te }\end{array}$} & $\begin{array}{l}\mathrm{ING}_{-} \\
\mathrm{MEC}\end{array}$ & 7.5 & 1.1 & \multirow{3}{*}{.595} \\
\hline & SC_EF & 7.1 & 2.2 & \\
\hline & SC_SOC & 8.0 & 1.9 & \\
\hline \multirow{3}{*}{$\begin{array}{l}\text { In che grado valuti la disponi- } \\
\text { bilità dei Tutor Junior }\end{array}$} & $\begin{array}{l}\mathrm{ING}_{-} \\
\mathrm{MEC}\end{array}$ & 7.7 & 1.5 & \multirow{3}{*}{.913} \\
\hline & SC_EF & 7.7 & 2.6 & \\
\hline & SC_SOC & 8.1 & 2.1 & \\
\hline \multirow{3}{*}{$\begin{array}{l}\text { In che misura ritieni ade- } \\
\text { guati gli orari di apertura } \\
\text { dell'Ufficio Tutorato }\end{array}$} & $\begin{array}{l}\mathrm{ING}_{-} \\
\mathrm{MEC}\end{array}$ & 6.7 & 1.2 & \multirow{3}{*}{.608 } \\
\hline & SC_EF & 6.0 & 1.9 & \\
\hline & SC_SOC & 6.8 & 1.3 & \\
\hline \multirow{3}{*}{$\begin{array}{l}\text { In che misura ritieni utile la } \\
\text { pagina web dei Tutor Junior } \\
\text { nel sito del tuo Corso di } \\
\text { Studio }\end{array}$} & $\begin{array}{l}\mathrm{ING}_{-} \\
\mathrm{MEC}\end{array}$ & 8.0 & 2.8 & \multirow{3}{*}{.853} \\
\hline & SC_EF & 6.7 & 3.0 & \\
\hline & SC_SOC & 7.0 & 1.7 & \\
\hline
\end{tabular}

4 I Tutor Junior sono studenti neo-laureati, laureandi o dottorandi con un percorso accademico particolarmente brillante, ai quali viene affidato il compito di facilitare I'inserimento delle matricole nel sistema universitario e di sostenere gli studenti in difficoltà degli anni successivi. 
Abandono, rendimiento académico y tutoría: una investigación de la Universidad de Padua

Lorenza Da Re y Renata Clerici

\begin{tabular}{|c|c|c|c|c|}
\hline Servizio Tutorato & $\mathrm{CdS}$ & $\begin{array}{c}\text { Soddisfazione } \\
\text { media }\end{array}$ & $\begin{array}{l}\text { Dev. } \\
\text { Stand. }\end{array}$ & Significatività \\
\hline \multirow{3}{*}{$\begin{array}{l}\text { In che misura hai gradito le } \\
\text { modalità di contatto (ad esem- } \\
\text { pio: mail, telefono, in presen- } \\
\text { za) utilizzate dai Tutor Junior }\end{array}$} & $\begin{array}{l}\mathrm{ING}_{-} \\
\mathrm{MEC}\end{array}$ & 8.7 & 1.9 & \multirow{3}{*}{.449} \\
\hline & SC_EF & 7.2 & 2.3 & \\
\hline & SC_SOC & 8.2 & 2.1 & \\
\hline \multirow{3}{*}{$\begin{array}{l}\text { Nel complesso, in che misura } \\
\text { sei soddisfatto del Servizio } \\
\text { Tutorato }\end{array}$} & $\begin{array}{l}\mathrm{ING}_{-} \\
\mathrm{MEC}\end{array}$ & 7.0 & 2.0 & \multirow{3}{*}{.865} \\
\hline & SC_EF & 7.5 & 2.3 & \\
\hline & SC_SOC & 7.6 & 1.5 & \\
\hline
\end{tabular}

Se consideriamo i risultati ottenuti con riferimento all'indicatore utilizzato per identificare il profilo di rendimento accademico degli studenti, il 44\% è classificato come "realista e fiducioso", il 9\% "rassegnato", I'11\% "pessimista", il 21\% "ottimista e realista" e il 14\% "ottimista ma non realista". La tabella 4 mette in evidenza alcune differenze tra i CdS, in particolare gli studenti di SC_SOC appaiono maggiormente "realisti fiduciosi" (61\%), soprattutto rispetto agli studenti di ING_MEC (35\%).

Tabella 4. Percentuali dei rispondenti dei CdS per rendimento accademico.

\begin{tabular}{ccccc}
\hline Performance e aspettative & ING_MEC & SC_EF & SC_SOC & Totale \\
\hline Ottimisti realisti & $25.5 \%$ & $21.1 \%$ & $14.3 \%$ & $21.3 \%$ \\
Realisti fiduciosi & $35.3 \%$ & $45.1 \%$ & $60.7 \%$ & $44.7 \%$ \\
Ottimisti non realisti & $13.7 \%$ & $15.5 \%$ & $10.7 \%$ & $14 \%$ \\
Rassegnati & $11.8 \%$ & $8.5 \%$ & $7.1 \%$ & $9.3 \%$ \\
Pessimisti & $13.7 \%$ & $9.9 \%$ & $7.1 \%$ & $10.7 \%$ \\
Totale & $100 \%$ & $100 \%$ & $100 \%$ & $100 \%$ \\
\hline
\end{tabular}

Di seguito presenteremo alcuni risultati, sintetizzati nella tabella 5; dalle analisi emerge che alcuni indicatori, utilizzati per rispondere alle varie domande di ricerca, risultano avere un legame particolarmente significativo con lo status dello studente alla fine del primo anno di corso associato alla sua percezione e alle sue aspettative rispetto all'esito che avrà la sua carriera universitaria, definibile come "outcome di carriera", o "rendimento accademico". A seconda del tipo di variabili considerate per verificarne l'influenza sull'outcome, sono state diverse le procedure statistiche applicate per la verifica della significatività delle relazioni. Si sono utilizzati test Chi Quadro per variabili qualitative e test F per con- 
fronto tra medie. In ogni caso le considerazioni sono state svolte in relazione alla presenza o meno di livelli significativi $\left(p_{-}\right.$value $\left.<.05\right)$ o molto significativi $\left(p_{-}\right.$value $\left.<.01\right)$ dei diversi test utilizzati.

In riferimento alla prima domanda, se alcune caratteristiche sociodemografiche influenzano il rendimento accademico, emerge che tra gli studenti di SC_EF vi è una propensione significativamente più elevata dei maschi a cambiare CdS rispetto alle femmine, e degli studenti residenti fuori regione ad abbandonare l'università.

In riferimento alla seconda domanda di ricerca, relativa all'influenza della condizione socio-economica sul rendimento accademico, si osserva che chi svolge attività part-time o a tempo pieno nel CdS in ING_MEC tende a essere più "regolare pessimista" e ad abbandonare di più, rispetto a chi non lavora o svolge attività di tipo precario o subordinato.

Se consideriamo la terza domanda di ricerca, ossia se "alcuni fattori psicologici legati allo studio influenzano il rendimento accademico", la capacità di studio risulta essere significativa per il CdS in SC_SOC, in cui la media di tale indice risulta più bassa per i "regolari pessimisti" e per coloro che cambiano percorso, mentre è significativamente più alta per i "regolari fiduciosi". In riferimento alla "motivazione", a ING_MEC i "regolari pessimisti", chi rallenta e chi cambia corso hanno una capacità di studio mediamente più bassa. A Scienze dell'Educazione e della Formazione è, invece, chi abbandona a presentare un indice di motivazione decisamente più basso.

Per la quarta domanda di ricerca, che indaga influenza dell'orientamento e della motivazione alla scelta nel rendimento accademico, e la quinta domanda, che valuta l'influenza del percorso pre-universitario sul rendimento accademico, non risultano evidenze significative.

Se consideriamo la sesta domanda, se alcuni fattori contestuali relativi al sistema universitario influenzano il percorso di studio, risulta significativa la residenza fuori sede per il CdS in SC_EF, dove si osserva una tendenza a rallentate maggiormente gli studi.

La settima domanda di ricerca, che ipotizza che conoscere e utilizzare i servizi universitari sia predittivo del successo accademico, trova riscontro significativo per il CdS in ING_MEC, ma limitatamente ad alcuni Servizi: Stage e Mondo del Lavoro (.000), Relazioni Internazionali (.005), Biblioteche (.025), Diritto allo Studio (.038).

L'ottava domanda, se la soddisfazione rispetto al percorso che si svolge sia predittiva del successo o dell'insuccesso, e la nona, se alcune 
Abandono, rendimiento académico y tutoría: una investigación de la Universidad de Padua

Lorenza Da Re y Renata Clerici

questioni legate alla didattica influiscano sul percorso di studio, non mostrano evidenze significative.

Infine, la decima domanda di ricerca, ossia se "alcune questioni legate alla docenza influiscono sul percorso di studio" evidenzia delle significatività per ING_MEC; nello specifico per la variabile "I docenti hanno stimolato/motivato l'interesse verso la loro disciplina" (.027): i "regolari fiduciosi" danno infatti una valutazione media più elevata rispetto al totale. É, inoltre, significativa per SC_EF la variabile "I docenti hanno svolto una funzione di supporto, indirizzo e accompagnamento al tuo percorso di studio" (.011): i "regolari fiduciosi" danno, infatti, anche in questo caso, una valutazione media più alta. Ancora per SC_EF risulta significativa anche la variabile "I docenti sono stati reperibili nell'orario di ricevimento per chiarimenti e spiegazioni" (.020): i "regolari pessimisti" e chi cambia CdS danno una valutazione più bassa rispetto alla media generale, mentre i "regolari fiduciosi" danno una valutazione più alta

\section{Tabella 5. Sintesi dei fattori risultati significativi rispetto alle domande di ricerca per $\mathrm{CdS}$.}

Domanda di ricerca e Significatività del test utilizza-

variabile significativa to per Corso di Studio

Note

in almeno un CdS ING_MEC SC_EF SC_SOC

(A) Fattori individuali (studente)

$\begin{array}{lcccl}\text { DR1: Residenza } & \text { n.s. } & .017 & \text { n.s. } & \begin{array}{l}\text { Cambiano CdS in mi- } \\ \text { sura maggiore gli stu- } \\ \text { denti di SC_EF }\end{array} \\ \text { DR1: Età } & \text { n.s. } & \text { n.s. } & \text { n.s. } & \\ \text { DR1: Sesso } & \text { n.s. } & .026 & \text { n.s. } & \begin{array}{l}\text { Più studenti maschi } \\ \text { cambiano Corso per il } \\ \text { CdS in SC_EF }\end{array}\end{array}$

DR1: Scuola di prove- n.s. n.s. n.s.

nienza

DR2: Esperienza uni- n.s. n.s. n.s. versitaria in famiglia

DR2: Condizione

$.052 \quad$ n.s. n.s.

Gli studenti che svolgono attività part time $\mathrm{O}$ a tempo pieno tendono a essere più "regolari pessimisti" e abbandonano di più nel $\mathrm{CdS}$ in ING_MEC 


\begin{tabular}{|c|c|c|c|c|}
\hline \multirow{2}{*}{$\begin{array}{c}\text { Domanda di ricerca e } \\
\text { variabile significativa } \\
\text { in almeno un } C d S\end{array}$} & \multicolumn{3}{|c|}{$\begin{array}{c}\text { Significatività del test utilizza- } \\
\text { to per Corso di Studio }\end{array}$} & \multirow[t]{2}{*}{ Note } \\
\hline & ING_MEC & $S C_{-} E F$ & SC_SOC & \\
\hline $\begin{array}{l}\text { DR2: Benefici econo- } \\
\text { mici }\end{array}$ & n.s. & n.s. & n.s. & \\
\hline $\begin{array}{l}\text { DR3: Capacità di } \\
\text { studio }\end{array}$ & n.s. & n.s. & .025 & $\begin{array}{l}\text { La media risulta essere } \\
\text { più bassa per SC_SOC, } \\
\text { per i "regolari pessi- } \\
\text { misti" e per coloro che } \\
\text { cambiano CdS, mentre } \\
\text { risulta essere più alta } \\
\text { per i "regolari fiduciosi" }\end{array}$ \\
\hline DR3: Motivazione & .010 & .012 & n.s. & $\begin{array}{l}\text { I "regolari pessimisti", } \\
\text { chi rallenta e chi cam- } \\
\text { bia CdS ha una media } \\
\text { più bassa del totale per } \\
\text { ING_MEC } \\
\text { Chi abbandona ha un } \\
\text { indice di motivazione } \\
\text { decisamente più bas- } \\
\text { So rispetto al totale per } \\
\text { SC_EF }\end{array}$ \\
\hline $\begin{array}{l}\text { DR4: Scelta consape- } \\
\text { vole e ragionata }\end{array}$ & n.s. & n.s. & n.s. & \\
\hline $\begin{array}{l}\text { DR5: Regolarità } \\
\text { Percorso pre-univer- } \\
\text { sitario }\end{array}$ & n.s. & n.s. & n.s. & \\
\hline DR5: Voto di diploma & n.s. & n.s. & n.s. & \\
\hline \multicolumn{5}{|c|}{ (B) Fattori del contesto (sistema universitario) } \\
\hline $\begin{array}{l}\text { DR6: Sede di studio e } \\
\text { domicilio }\end{array}$ & n.s. & .022 & n.s. & $\begin{array}{l}\text { Chi cambia CdS a } \\
\text { SC_EF risiedeva o era } \\
\text { domiciliato nella pro- } \\
\text { vincia del CdS, chi non } \\
\text { risiede o non è domici- } \\
\text { liato nella provincia del } \\
\text { CdS tende a rallentate } \\
\text { maggiormente gli studi }\end{array}$ \\
\hline $\begin{array}{l}\text { DR6: Frequenza } \\
\text { lezioni }\end{array}$ & n.s. & n.s. & n.s. & \\
\hline $\begin{array}{l}\text { DR7: Conoscenza/ } \\
\text { utilizzo tutorato }\end{array}$ & n.s. & n.s. & n.s. & \\
\hline
\end{tabular}


Abandono, rendimiento académico y tutoría: una investigación de la Universidad de Padua

Lorenza Da Re y Renata Clerici

\begin{tabular}{|c|c|c|c|c|}
\hline \multirow{2}{*}{$\begin{array}{c}\text { Domanda di ricerca e } \\
\text { variabile significativa } \\
\text { in almeno un } \mathrm{CdS}\end{array}$} & \multicolumn{3}{|c|}{$\begin{array}{c}\text { Significatività del test utilizza- } \\
\text { to per Corso di Studio } \\
\end{array}$} & \multirow[t]{2}{*}{ Note } \\
\hline & ING_MEC & $S C \_E F$ & SC_SOC & \\
\hline $\begin{array}{l}\text { DR7: Conoscenza dei } \\
\text { Servizi }\end{array}$ & $\begin{array}{l}.000 \\
.005 \\
.025 \\
.038\end{array}$ & n.s. & n.s. & $\begin{array}{l}\text { II Test F risulta essere } \\
\text { significativo per ING_ } \\
\text { MEC solamente per } \\
\text { alcuni Servizi: Servizio } \\
\text { Stage e Mondo del La- } \\
\text { voro (.000), Servizio } \\
\text { Relazioni Internazio- } \\
\text { nali (.005), Biblioteche } \\
\text { (.025), Servizio Diritto } \\
\text { allo Studio (.038) }\end{array}$ \\
\hline $\begin{array}{l}\text { DR8: Soddisfazione } \\
\text { rapporti }\end{array}$ & n.s. & n.s. & n.s. & \\
\hline $\begin{array}{l}\text { DR8: Soddisfazione } \\
\text { CdS }\end{array}$ & n.s. & n.s. & n.s. & \\
\hline $\begin{array}{l}\text { DR8: Soddisfazione } \\
\text { generale }\end{array}$ & n.s. & n.s. & n.s. & \\
\hline
\end{tabular}

(C) Fattori del contesto (didattica e docenza)

DR9: Valutazione

n.s.

n.s.

n.s.

della didattica

DR10: Valutazione

$.027 \quad .011$

.020

n.s. Risulta essere signifidella docenza cativa per ING_MEC la variabile "I docenti hanno stimolato/motivato l'interesse verso la loro disciplina" (.027): $i$ "regolari fiduciosi" danno una valutazione media più elevata rispetto al totale. I "regolari fiduciosi" danno infatti una valutazione media $(6,24)$ più elevata rispetto al totale.

É significativa per SC_EF la variabile "I docenti hanno svolto una funzione di supporto, indirizzo e accompagnamento al tuo 


\begin{tabular}{|c|c|c|}
\hline \multirow{2}{*}{$\begin{array}{c}\text { Domanda di ricerca e } \\
\text { variabile significativa } \\
\text { in almeno un CdS }\end{array}$} & $\begin{array}{c}\text { Significatività del test utilizza- } \\
\text { to per Corso di Studio }\end{array}$ & \multirow[t]{2}{*}{ Note } \\
\hline & ING_MEC SC_EF SC_SOC & \\
\hline & & $\begin{array}{l}\text { percorso di studio" } \\
\text { (.011), i "regolari fidu- } \\
\text { ciosi" danno infatti una } \\
\text { valutazione media più } \\
\text { alta. Risulta significa- } \\
\text { tiva anche la variabile } \\
\text { "I docenti sono stati } \\
\text { reperibili nell'orario di } \\
\text { ricevimento per chia- } \\
\text { rimenti e spiegazioni" } \\
\text { (.O20): in questo caso } \\
\text { i "regolari pessimisti" e } \\
\text { chi cambia CdS danno } \\
\text { una valutazione più } \\
\text { bassa rispetto alla me- } \\
\text { dia generale, mentre } i \\
\text { "regolari fiduciosi" dan- } \\
\text { no una valutazione più } \\
\text { alta. }\end{array}$ \\
\hline
\end{tabular}

\section{Conclusioni e prospettive future}

Obiettivo dell'indagine era di analizzare i fattori influenti sui fenomeni connessi alla dispersione nei percorsi universitari nei diversi contesti scientifico/didattici, per poi poter attivare o potenziare interventi di tutorato, tenendo conto proprio di tali specificità. Questo obiettivo può dirsi raggiunto, in quanto le analisi condotte hanno permesso sia di ricavare importanti informazioni sul modo in cui gli studenti si approcciano al mondo universitario, sia perché, a partire da una profilazione degli studenti in termini sia oggettivi (performance accademica in termini di crediti conseguiti), che in termini soggettivi (la percezione che ne hanno al termine del primo anno di università), si sono potute affrontate una serie di domande di ricerca circa l'influenza di quei fattori personali e contestuali che la letteratura segnala come di particolare rilevanza nell'interpretazione dei fenomeni della dispersione universitaria.

Dai dati presentati emergono delle differenze rilevanti nei tre contesti analizzati: 
Abandono, rendimiento académico y tutoría: una investigación de la Universidad de Padua

Lorenza Da Re y Renata Clerici

- Per quanto riguarda gli studenti di Scienze dell'Educazione e della Formazione, essi cambiano CdS in misura maggiore, e i maschi in particolare. Chi abbandona presenta un indice di motivazione decisamente più basso. Gli studenti che non risiedono o non sono domiciliati nella provincia della sede universitaria tendono a rallentate maggiormente gli studi. É, inoltre, significativa la variabile "I docenti hanno svolto una funzione di supporto, indirizzo e accompagnamento al tuo percorso di studio": i "regolari fiduciosi" di questo CdS danno, infatti, una valutazione media più alta. Risulta significativa anche la variabile "I docenti sono stati reperibili nell'orario di ricevimento per chiarimenti e spiegazioni": in questo caso i "regolari pessimisti" e chi cambia CdS danno una valutazione più bassa rispetto alla media generale, mentre i "regolari fiduciosi" danno una valutazione più alta.

- Tra gli studenti di Ingegneria Meccanica chi svolge attività parttime o a tempo pieno tende a essere più "regolare pessimista" e ad abbandonare di più. Tra gli studenti "regolari pessimisti", chi rallenta e chi cambia $\mathrm{CdS}$ ha una motivazione media relativamente più bassa. Per quanto riguarda i fattori di contesto, risulta essere significativa per questo CdS la conoscenza di alcuni Servizi (Stage e Mondo del Lavoro, Relazioni Internazionali, Biblioteche, Diritto allo Studio). Risultano anche essere significative alcune variabili relative a questioni legate alla docenza che influiscono sul percorso di studio: nello specifico, per la variabile "I docenti hanno stimolato/motivato l'interesse verso la loro disciplina", i "regolari fiduciosi" danno una valutazione media più elevata rispetto al totale.

- Per gli studenti di Scienze Sociologiche la "capacità di studio" risulta essere significativa: la media di tale indice risulta più bassa per i "regolari pessimisti" e per coloro che cambiano CdS, mentre è più alta per i "regolari fiduciosi".

A partire dall'anno accademico 2014-15 I'Università di Padova ha implementato le azioni di tutorato con il Programma di Tutorato Formativo. Il Programma consiste in attività di supporto agli studenti del primo anno di alcuni CdS triennali dell'Università di Padova, e si realizza attraverso incontri settimanali di un'ora di: (i) Tutorato dei Servizi, riunioni informative in plenaria sui Servizi universitari; (ii-iii) Incontri con i Tutor Docenti 
e Tutor Studenti, in cui gli studenti lavorano in piccolo gruppo sulle competenze trasversali attraverso il supporto e coordinamento di un docente del CdS e/o di uno studente del secondo o terzo anno del CdS.

Dalla sperimentazione (cfr. Da Re, 2016) emerge che i tre CdS analizzati hanno risposto in maniera differente, dimostrando che il modello, per poter funzionare, dev'essere calato nel contesto specifico e deve adattarsi, anno per anno, alle necessità degli studenti e alle caratteristiche dei tutor (docenti e studenti) disponibili.

Ad oggi la sperimentazione del Tutorato Formativo è stata ampliata anche a altri CdS triennali dell'Università di Padova (Astronomia, Fisica, Ingegneria Biomedica, Ingegneria Meccanica, Ottica e Optometria, Scienze dell'Educazione e della Formazione, Servizio Sociale, Scienze Sociologiche) grazie al Progetto di Ateneo "Tutorato Formativo: un modello di tutoring integrato per l'empowerment degli studenti universitari".

Indagini analoghe a quella qui presentata, costituiscono per le nuove sperimentazioni una base di conoscenza delle specificità dei contesti di intervento che possono porre le corrette premesse alla progettazione di azioni adeguate ed efficaci.

\section{Referencias bibliográficas}

Albalate, D., Fageda X., e Perdiguero J. (2011). Éxito Académico, Características Personales y Proceso de Bolonia: Una Aplicación Econométrica. Revista d'Innovació Docent Universitària, 3, 11-25.

Álvarez, P. (2002). La función tutorial en la universidad: Una apuesta por la mejora de la calidad de la enseñanza. Madrid: EOS.

Arias Ortiz, E. A., e Dehon, C. (2013). Roads to Success in the Belgian French Community's Higher Education System: Predictors of Dropout and Degree Completion at the Université Libre de Bruxelles. Research in Higher Education, 54, 693-723.

Arulampalam, W., Naylor, R. A., e Smith, J.P. (2004). A hazard model of the probability of medical school dropout in the UK. Journal of the Royal Statistical Society. Series A, $167,1,157-178$.

Barefoot, B. (2004). Higher education's revolving door: confronting the problem of student drop out in US colleges and universities. Open Learning, 19 (1), 9-18.

Belloc, F., Maruotti, A., e Petrella, L. (2010). University drop-out: an Italian experience. Higher education, 60, 127-138.

Berger, J. B. (2002). Understanding the organizational nature of student persistence empirically-based recommendations for practice. Journal of College Student Retention: Research, Theory and Practice, 3 (1), 3-21. 
Abandono, rendimiento académico y tutoría: una investigación de la Universidad de Padua

Lorenza Da Re y Renata Clerici

Braunstein, A., Mc Grath, M., e Pescatrice, D. (2001). Measuring the impact of financial factors on college persistence. Journal of College Student Retention, 2 (3), 191-203.

Braxton, J. M., e Mcclendon, S. A. (2002). The fostering of social integration and retention through institutional practice. Journal of College Student Retention, 3 (1), 57-71.

Charlton, J. P., Barrow, C., e Atkinson, H. (2006). Attempting to predict withdrawal from higher education using demographic, psychological and educational measures. Research in Post Compulsory Education, 11 (1), 31-47.

Chen, R. (2012). Institutional characteristics and college student dropout risks: A multilevel event history analysis. Research in Higher education, 53, 487-505.

Clerici, R., Giraldo, A., e Meggiolaro, S. (2015). The determinants of academic outcomes in a competing risks approach: evidence from Italy. Studies in Higher Education, 40 (9), 1535-1549. DOI: 10.1080/03075079.2013.878835.

Cofer, J., e Somers, P. (2001). What influences student persistence at two-year colleges? A multi-year comparison. Community College Review, 29 (3), 56-76.

Corominas, E. (2001). La transición a los estudios universitarios. Abandono o cambio en el primer año de universidad. Revista de Investigación Educativa, 19 (1), 127-151.

Da Re, L. (2016). Il Tutorato Formativo come strategia per la prevenzione del drop-out e per il miglioramento del rendimento accademico degli studenti universitari. La Tutoría Formativa como estrategía para la prevención del abandono y la mejora del rendimiento académico en estudiantes universitarios. Tesi di Dottorato in Scienze Pedagogiche dell'Educazione e della Formazione, Università degli Studi di Padova; Escuela de Educación, Università de La Laguna (ES), Supervisori Prof.ssa R. Clerici e Prof. P.R. Álvarez Pérez.

Da Re, L., Álvarez Pérez, P., e Clerici, R. (2015). Adaptación al contexto universitario italiano del modelo de tutoría formativa para la prevención del abandono y la mejora del rendimiento académico. En AIDIPE (Ed.), Investigar con y para la sociedad (Vol. 2), pp. 721-730. Cádiz, España: Bubok.

Da Re, L., Alvarez, P., e Clerici. R. (2016). Le attività e gli strumenti del Programma di Tutorato Formativo per i nuovi iscritti all'Università: una guida operativa. Padova: Cleup.

Da Re, L., e Zago, G. (2014). Il tutorato a Padova: caratteristiche e risultati d'indagine, In G. Zago, A. Giraldo, e Clerici R. (A cura di), Carriere universitarie tra successo e insuccesso. Dati, interpretazioni e proposte. Bologna: il Mulino.

Desjardins, S. L., Ahlburg, D. A., e Mccall, B. (2002). A temporal investigation of factors related to timely degree completion. The Journal of Higher Education, 73 (5), 555581.

Dolton, P., Marcenaro, O. D., e Navarro, L. (2003). The effective use of student time: A stochastic frontier production function case study. Economics of Education Review, 22, 547-560.

Escandell, O., e Marrero, G. (1999). El abandono de los estudiantes en la universidad de Las Palmas de Gran Canaria: sus causas, propuestas de estrategias para su disminución. Las Palmas de Gran Canaria: La Caja de Canarias.

Fabbris, L. (2010). I servizi universitari. La proposta degli stakeholder. Padova: Cleup.

Forbes, A., eE Wickens, E. (2005). A good social life helps students to stay the course. Times Higher Education Supplement. Sigue, 1676, 58-63. 
Abandono, rendimiento académico y tutoría: una investigación de la Universidad de

Padua

lorenza Da Re y Renata Clerici

García Félix, E., Conejero Casares, J. A., e Díez Ruano, J. L. (2014). La entrada en la Universidad: un reto para la orientación académica. Revista De Docencia Universitaria, 12 (2), 255-280.

Ishitani, T., e Desjardins, S. L. (2002). A longitudinal investigation of dropout from college in the United States. Journal of College Student Retention: Research, Theory \& Practice, 4 (2), 173-201.

Kim, D. B. (2007). The effect of loans on students' degree attainment: Differences by student and institutional characteristics. Harvard Educational Review, 77 (1), 64-100.

Kirton, M. J. (2000). Transitional factors influencing the academic persistence of first semester undergraduate freshmen. Dissertation Abstracts International Section A: $\mathrm{Hu}$ manities and Social Sciences, 61 (2-A), 522.

Kuh, G. D. (2002). Organizational culture and student persistence: prospects and puzzles. Journal of College Student Retention. Research, Theory \& Practice, 3 (1), 23-39.

Landry, C. (2003). Self-efficacy, motivation and outcome expectation correlates of college students' intention certainty. Dissertation Abstracts International Section A: Humanities and Social Sciences, 64 (3-A), 825.

Latiesa, M. (1992). La deserción universitaria. Madrid: Centro de Investigaciones sociológicas.

Lyons, A. (2004). A profile of financially at-risk college students. The Journal of consumer affairs, 38 (1), 56-80.

Martìnez Clares, P., Pérez Cusò, J., e Martìnez Juàrez, M., (2014). Una (re)visión de la tutoría universitaria en los estudios de Grado. REDU, 12 (2), 269-305.

Mashburn, A. J. (2000). A psychological process of student dropout. Journal of College Student Retention, Research, Theory \& Practice, 2 (3), 173-190.

Masjoan, J. M. (1989). Escala de valores instrumentales, autoestima y permanencia en el sistema educativo. Sociología de la Educación, 11, 169-203.

Meggiolaro, S., Giraldo, A., e Clerici, R. (2015). A multilevel competing risks model for analysis of university students' careers in Italy. Studies in higher education, DOI: 10.1080/03075079.2015.1087995.

Metz, G. W. (2002). Challenges and changes to Tinto's persistence theory. Paper presented at the Annual Meeting of the Mid-Western Educational Research Association. Columbus, $\mathrm{OH}$, October 16-19.

Montmarquette, C., Mahseredjian, S., e Houle, R., (2001). The determinants of University dropouts: a bivariate probability model with sample selection'. Economics of Education Review, 20 (5), 474-84.

Moxley, D. M., Najor-Durack, A., e Dumbrigue, C. (2001). Keeping Students in Higher Education: successful practices \& strategies for retention. England: Kogan Page Limited.

O’Neill, L. D., Wallstedt, B., Eika, B., e Hartvigsen, J. (2011). Factors associated with dropout in medical education: A literature review. Medical Education, 45, 440-454.

Pantoja, A. (2005). La acción tutorial en la universidad: propuestas para el cambio, Cultura y Educación. Culture and Education, 17 (1), 67-82.

Patrick, W. J. (2001). Estimating first-year student attrition rates: An application of multilevel modelling using categorical variables. Research in Higher education, 42 (2), 151-170. 
Abandono, rendimiento académico y tutoría: una investigación de la Universidad de

Padua

Lorenza Da Re y Renata Clerici

Pérez, F. C., Martínez, P. C., e Martínez, M. J. (2015). Satisfacción del estudiante universitario con la tutoría. Diseño y validación de un instrumento de medida. Estudios sobre educación. 29, 81-101. DOI: 10.15581/004.29.81-101.

Reason, R. D. (2009). Student variables that predict retention: Recent research and new developments. Journal of Student Affairs Research and Practice, 46 (3), 482-501.

Ryan J. F. (2004). The relationship between institutional expenditures and degree attainment at baccalaureate colleges. Research in Higher Education, 45 (2), 97-114.

Seligman, M. E. (1990). Learned optimist. New York: Pocket Books.

Sinclair, H., e Dale, T. (2000). The effect of student tuition fees on the diversity of intake within a scottish new university. Paper presented at British Educational Research Association Annual Conference, 7-9 September, 2000, Cardiff University.

Smith, J. P., e Naylor, R. A. (2001). Dropping out of university: A statistical analysis of the probability of withdrawal for UK university students. Journal of the Royal Statistical Society-Series A, 164, 389-405.

Snyder, C. R. (2002). Hope theory: rainbows in the mind. Psychological Inquiry, 13 (4), 249-275.

Soria-Barreto, K., e Zúñiga-Jara, S. (2014). Determining aspects of academic success of University students. Formación universitaria, 7 (5), 41-50.

Spady, W. (1970). Dropouts from higher education: an interdisciplinary review and synthesis. Interchange. 1, 64-85.

Thomas, L. (2002). Student retention in higher education: the role of institutional habitus. Journal of Education Policy, 17 (4), 423-442.

Tinto, V. (1975). Dropout from higher education: A theoretical synthesis of recent research. Review of Educational Research, 45, 89-125.

Titus, M. A. (2004). An examination of the influence of institutional context on student persistence at 4-year colleges and universities: A multilevel approach. Research in Higher Education. 45 (7), 673-699.

Zabalza, M. (2003). Competencias docentes del profesorado universitario: calidad y desarrollo profesional. Madrid: Narcea.

Zago, G., Girado, A., e Clerici, R. (A cura di). (2014). Successo e insuccesso negli studi universitari. Bologna: II Mulino.

Zimmerman, B. J. (2000). Attaining self-regulation: A social cognitive perspective, in $\mathrm{M}$. Boekaerts, P. R. Pintrich, e M. Zeidner (A cura di), Handbook of Self-Regulation (pp. 13-39). San Diego, Calif, USA: Academic Press.

Zubieta, J. C., e Susinos, F. (1986). Desigualdad de formación y rendimiento académico en las escuelas universitarias. In M. Latiesa, Demanda de educación superior y rendimiento académico en la universidad. Madrid: MEC. 